\title{
Resistance matrices of graphs with matrix weights
}

\author{
Fouzul Atik* $\quad$ R. B. Bapat ${ }^{\dagger} \quad$ M. Rajesh Kannan ${ }^{\ddagger}$
}

April 5, 2018

\begin{abstract}
The resistance matrix of a simple connected graph $G$ is denoted by $R$, and is defined by $R=\left(r_{i j}\right)$, where $r_{i j}$ is the resistance distance between the vertices $i$ and $j$ of $G$. In this paper, we consider the resistance matrix of weighted graph with edge weights being positive definite matrices of same size. We derive a formula for the determinant and the inverse of the resistance matrix. Then, we establish an interlacing inequality for the eigenvalues of resistance and Laplacian matrices. Using this interlacing inequality, we obtain the inertia of the resistance matrix.
\end{abstract}

Keywords. Resistance matrix, Laplacian matrix, Matrix weighted graph, Inverse, Inertia, MoorePenrose inverse.

2010 Mathematics Subject Classification: 05C50.

\section{Introduction}

Consider an $n$-vertex connected graph $G=(V, E)$, where $V=\{1,2, \ldots, n\}$ is the vertex set and $E=\left\{e_{1}, e_{2}, \ldots, e_{m}\right\}$ is the edge set. If two vertices $i$ and $j$ of $G$ are adjacent, we denote it by $i \sim j$. A (positive scalar) weighted graph is graph in which every edge is assigned a weight which is a positive number. If weight of the each edge is 1 , the graph is called an unweighted graph. The weight of a path is the sum of the weights of the edges lies in the path. The distance matrix $D(G)$ of a weighted graph $G$ is an $n \times n$ matrix $\left(d_{i j}\right)$, where $d_{i j}$ is the minimum of weights of all paths from the vertex $i$ to the vertex $j$. The Laplacian matrix $L$ of a weighted graph $G$ is an $n \times n$ matrix defined as follows: For $i, j \in[n]=\{1, \ldots, n\}, i \neq j$, the $(i, j)^{t h}$-element of $L$ is $-\frac{1}{w_{i j}}$ if the vertices $i$ and $j$ are adjacent, where $w_{i j}$ denotes the weight of the edge joining the vertices $i$ and $j$, and it is zero, otherwise. For $i \in[n]$, the $(i, i)^{t h}$-entry of the matrix $L$ is $\sum_{j \sim i} \frac{1}{w_{i j}}$. Let $e_{i j}$ denote the $n \times 1$ vector with 1 at the $i^{t h}$ place, -1 at the $j^{\text {th }}$ place, and zero elsewhere. A matrix $H$ is called a generalized inverse, or $g$-inverse of $L$ if $L H L=L$. It is known [4] that for a graph with positive edge weights, the scalar $e_{i j}^{\prime} H e_{i j}$ is same for any $g$-inverse $H$ of $L$ where $e_{i j}^{\prime}$ is the transpose of $e_{i j}$. The resistance distance between the vertices $i$ and $j$, denoted by $r(i, j)$, defined as $r(i, j)=e_{i j}^{\prime} H e_{i j}=h_{i i}+h_{j j}-h_{i j}-h_{j i}$, where $H$ is a $g$-inverse of $L$. In particular, if $L^{\dagger}=\left(k_{i j}\right)$ is the Moore-Penrose inverse(defined in Section 2) of $L$, then

\footnotetext{
${ }^{*}$ Theoretical Statistics and Mathematics Unit, Indian Statistical Institute, New Delhi 110 016, India. Email: fouzulatik@gmail.com

${ }^{\dagger}$ Theoretical Statistics and Mathematics Unit, Indian Statistical Institute, New Delhi 110 016, India. Email: rbb@isid.ac.in

${ }^{\ddagger}$ Department of Mathematics, Indian Institute of Technology Kharagpur, Kharagpur, India. Email: rajeshkannan@maths.iitkgp.ernet.in, rajeshkannan1.m@gmail.com
} 
$r(i, j)=k_{i i}+k_{j j}-2 k_{i j}$. The resistance matrix $R$ of a graph is an $n \times n$ matrix defined as follows: For $i, j \in[n]$, the $(i, j)^{t h}$-entry of $R$ is defined as $r_{i j}=r(i, j)$. For a tree, the resistance distance coincides with the classical distance.

The distance matrix of a connected graph, in particular of a tree, has been studied by researchers for many years. One of the remarkable result about the distance matrix of a tree states that, if $T$ is a tree on $n$ vertices, then the determinant of the distance matrix $D$ of $T$ is $(-1)^{n-1}(n-1) 2^{n-2}$, which is independent of the structure of the underlying tree [7]. In [6], a formula for the inverse of the distance matrix of a tree is obtained. An extension of these results for the weighted trees, where the weights are positive scalars, were considered in [5]. In [3], formulae for determinant and inertia of the distance matrix of a weighted tree with edge weights being square matrices of same order. Resistance distance matrices are one of the important classes of matrices, associated with graphs, considered in the chemical literature. The notion of Wiener index based on the resistance distance has proposed in [9]. The formulae for the determinant and the inverse of the resistance matrix of scalar weighted graph are obtained in [2]. Some inequalities for the eigenvalues of the distance matrix of a tree and the eigenvalues of the resistance matrix of any connected graph are established in [12] and [13], respectively. We refer to [10, 11, 15, 14, 16] for more information on the resistance distance.

In this paper, we consider the resistance matrix of any weighted graph whose edge weights are positive definite matrices of same size. We derive a formula for the determinant(Theorem 4.1) and the inverse(Theorem 4.2) of the resistance matrix. We establish an interlacing inequality for the eigenvalues of resistance and Laplacian matrices(Theorem 4.3). Using this, we obtain the inertia of the resistance matrix(Theorem 4.4).

This article is organized as follows: In section 2, we first recall some of the definitions and results, which will be used in the subsequent part of the paper. Then we establish two important properties, one of them related to the principal submatrix of Moore-Penrose inverse of a positive semidefinite $\operatorname{matrix}($ Lemma 2.1) and another is about the cofactor of block matrices(Lemma 2.2). In section 3 , we derive some of the properties of the resistance matrix with positive definite matrix edge weights. Using these properties, in section 4, we obtain formulae for the determinant, inverse and inertia of the resistance matrix with positive definite matrix edge weights.

\section{Some useful results}

Let $B$ be an $n \times n$ matrix and let $S, K \subseteq[n]$. We denote by $B[S, K]$, the matrix obtained by selecting the rows of $B$ indexed by $S$, and the columns of $B$ indexed by $K$. On the other hand, $B(S, K)$ is the matrix obtained by deleting the rows of $B$ indexed by $S$, and the columns of $B$ indexed by $K$. Similarly, if $x$ is an $n \times 1$ vector, then $x[S]$ will denote the subvector of $x$ indexed by indices in $S$. The Kronecker product of matrices $A=\left(a_{i j}\right)$ of size $m \times n$ and $B$ of size $p \times q$, denoted by $A \otimes B$, is defined to be the $m p \times n q$ block matrix $\left(a_{i j} B\right)$. It is known that for matrices $M, N, P$ and $Q$ of suitable sizes, $M N \otimes P Q=(M \otimes P)(N \otimes Q)[8]$. 
Let $A$ be an $n \times n$ matrix partitioned as

$$
A=\left[\begin{array}{ll}
A_{1,1} & A_{1,2} \\
A_{2,1} & A_{2,2}
\end{array}\right]
$$

such that the diagonal blocks $A_{1,1}$ and $A_{2,2}$ are square matrices and the matrix $A_{1,1}$ is invertible. Then the Schur complement of $A_{1,1}$ in $A$ is defined to be the matrix $A_{2,2}-A_{2,1} A_{1,1}^{-1} A_{1,2}$. The determinant of the matrix $A$ and determinant of the Schur complement of $A_{1,1}$ in $A$ has the following interesting relationship: $\operatorname{det} A=\operatorname{det}\left(A_{1,1}\right)\left(\operatorname{det} A_{2,2}-A_{2,1} A_{1,1}^{-1} A_{1,2}\right)$. This is called the Schur formula for the determinant.

Let $B^{\prime}$ denote the transpose of the matrix $B$. Let $A$ be an $m \times n$ matrix. A matrix $G$ of order $n \times m$ is said to be a generalized inverse (or a $g$-inverse) of $A$ if $A G A=A$. A matrix $G$ of order $n \times m$ is said to be the Moore-Penrose inverse of $A$ if it satisfies (i) $A G A=A$, (ii) $G A G=G$, (iii) $(A G)^{\prime}=A G$ and (iv) $(G A)^{\prime}=G A$. We denote the Moore-Penrose inverse of the matrix $A$ by $A^{\dagger}$.

In the next lemma, we prove that if a principal submatrix $A[S, S]$ of a positive semidefinite matrix $A$ is invertible, then the principal submatrix of $A^{\dagger}$ corresponds to the same index set $S$ is also invertible. i.e., $A^{\dagger}[S, S]$ is invertible.

Lemma 2.1. Let $A$ be an $n \times n$ positive semidefinite matrix, whose rows and columns are indexed by $[n]$. For $S \subseteq[n]$, if $A[S, S]$ is nonsingular then $A^{\dagger}[S, S]$ is also nonsingular.

Proof. Let $A$ be of rank $r(\leq n)$. For $i=1,2, \cdots, r$, let $\lambda_{i}$ be the nonzero eigenvalues of $A$ with corresponding eigenvectors $x_{i}$. Then the spectral decomposition of $A$ is given by

$$
A=\lambda_{1} x_{1} x_{1}^{\prime}+\lambda_{2} x_{2} x_{2}^{\prime}+\cdots+\lambda_{r} x_{r} x_{r}^{\prime} .
$$

Let $S \subseteq[n]$ with $|S|=p(\leq r)$ and $A[S, S]$ is nonsingular. Then

$$
\begin{aligned}
A[S, S] & =\lambda_{1} x_{1}[S] x_{1}^{\prime}[S]+\lambda_{2} x_{2}[S] x_{2}^{\prime}[S]+\cdots+\lambda_{r} x_{r}[S] x_{r}^{\prime}[S] \\
& =\left[\sqrt{\lambda_{1}} x_{1}[S] \sqrt{\lambda_{2}} x_{2}[S] \cdots \sqrt{\lambda_{r}} x_{r}[S]\right]\left[\begin{array}{c}
\sqrt{\lambda_{1}} x_{1}^{\prime}[S] \\
\sqrt{\lambda_{2}} x_{2}^{\prime}[S] \\
\cdots \\
\sqrt{\lambda_{r}} x_{r}^{\prime}[S]
\end{array}\right] .
\end{aligned}
$$

Since the rank of the matrix $A[S, S]$ is $p$, the rank of $\left[\sqrt{\lambda_{1}} x_{1}[S] \sqrt{\lambda_{2}} x_{2}[S] \cdots \sqrt{\lambda_{r}} x_{r}[S]\right]$ is also $p$. Again

$$
\left[\sqrt{\lambda_{1}} x_{1}[S] \sqrt{\lambda_{2}} x_{2}[S] \cdots \sqrt{\lambda_{r}} x_{r}[S]\right]=\left[x_{1}[S] x_{2}[S] \cdots x_{r}[S]\right]\left[\begin{array}{cccc}
\sqrt{\lambda_{1}} & 0 & \cdots & 0 \\
0 & \sqrt{\lambda_{2}} & \cdots & 0 \\
\cdots & \cdots & \ddots & \cdots \\
0 & 0 & \cdots & \sqrt{\lambda_{r}}
\end{array}\right] .
$$

Thus we get rank of $\left[x_{1}[S] x_{2}[S] \cdots x_{r}[S]\right]$ is $p$. Then rank of

$$
\left[x_{1}[S] x_{2}[S] \cdots x_{r}[S]\right]\left[\begin{array}{cccc}
\frac{1}{\sqrt{\lambda_{1}}} & 0 & \cdots & 0 \\
0 & \frac{1}{\sqrt{\lambda_{2}}} & \cdots & 0 \\
\cdots & \cdots & \ddots & \cdots \\
0 & 0 & \cdots & \frac{1}{\sqrt{\lambda_{r}}}
\end{array}\right]=\left[\frac{1}{\sqrt{\lambda_{1}}} x_{1}[S] \frac{1}{\sqrt{\lambda_{2}}} x_{2}[S] \cdots \frac{1}{\sqrt{\lambda_{r}}} x_{r}[S]\right]
$$


is also $p$. We note that the spectral decomposition of $A^{+}$is

$$
A^{\dagger}=\frac{1}{\lambda_{1}} x_{1} x_{1}^{\prime}+\frac{1}{\lambda_{2}} x_{2} x_{2}^{\prime}+\cdots+\frac{1}{\lambda_{r}} x_{r} x_{r}^{\prime} .
$$

Thus

$$
A^{\dagger}[S, S]=\frac{1}{\lambda_{1}} x_{1}[S, S] x_{1}^{\prime}[S, S]+\frac{1}{\lambda_{2}} x_{2}[S, S] x_{2}^{\prime}[S, S]+\cdots+\frac{1}{\lambda_{r}} x_{r}[S, S] x_{r}^{\prime}[S, S]
$$

is of rank $p$ and hence $A^{\dagger}[S, S]$ is also nonsingular.

Next we define the cofactor of a submatrix. Let $S=\left\{i_{1}, i_{2}, \cdots, i_{k}\right\}$ and $K=\left\{j_{1}, j_{2}, \cdots, j_{k}\right\}$ be subsets of $[n]$. For a matrix $A$ of order $n \times n$, cofactor of the submatrix $A[S, K]$ in $A$ is

$$
A_{S K}=(-1)^{i_{1}+i_{2}+\cdots+i_{k}+j_{1}+j_{2}+\cdots+j_{k}} \operatorname{det} A(S, K) .
$$

Consider a square matrix $A$ whose rows and columns are indexed by elements in $X=\{1,2, \cdots, n s\}$ and a partition $\pi=\left\{X_{1}, X_{2}, \cdots, X_{n}\right\}$ of $X$ where $\left|X_{i}\right|=s$ for all $i=1,2, \cdots, n$. We partition the matrix $A$ according to $\pi$ as

$$
A=\left[\begin{array}{cccc}
A_{1,1} & A_{1,2} & \cdots & A_{1, n} \\
A_{2,1} & A_{2,2} & \cdots & A_{2, n} \\
\cdots & \cdots & \cdots & \cdots \\
A_{n, 1} & A_{n, 2} & \cdots & A_{n, n}
\end{array}\right]
$$

where each $A_{i, j}=A\left[X_{i}, X_{j}\right]$ is a submatrix (block) of $A$ whose rows and columns are indexed by elements of $X_{i}$ and $X_{j}$, respectively.

Lemma 2.2. Let $A$ be an $n s \times n s$ square matrix and it is partitioned as in (3)). Let $\sum_{j=1}^{n} A_{i, j}=0$ and $\sum_{i=1}^{n} A_{i, j}=0$ for all $i, j \in\{1,2, \cdots, n\}$. Then the cofactor of any two blocks $A_{i, j}$ and $A_{k, l}$ in $A$ are equal. 
Proof. We have

$$
\begin{aligned}
\text { Cofactor of } A_{1,1}= & (-1)^{1+2+\cdots+s+1+2+\cdots+s} \operatorname{det}\left[\begin{array}{cccc}
A_{2,2} & A_{2,3} & \cdots & A_{2, n} \\
A_{3,2} & A_{3,3} & \cdots & A_{3, n} \\
\cdots & \cdots & \cdots & \cdots \\
A_{n, 2} & A_{n, 3} & \cdots & A_{n, n}
\end{array}\right] \\
= & \operatorname{det}\left[\begin{array}{cccc}
\sum_{j=2}^{n} A_{2, j} & A_{2,3} & \cdots & A_{2, n} \\
\sum_{j=2}^{n} A_{3, j} & A_{3,3} & \cdots & A_{3, n} \\
\cdots & \cdots & \cdots & \cdots \\
\sum_{j=2}^{n} A_{n, j} & A_{n, 3} & \cdots & A_{n, n}
\end{array}\right] \\
= & \operatorname{det}\left[\begin{array}{cccc}
-A_{2,1} & A_{2,3} & \cdots & A_{2, n} \\
-A_{3,1} & A_{3,3} & \cdots & A_{3, n} \\
\cdots & \cdots & \cdots & \cdots \\
-A_{n, 1} & A_{n, 3} & \cdots & A_{n, n}
\end{array}\right]\left[\begin{array}{lll}
\text { As } \\
A_{j=1}^{n}
\end{array} A_{i, j}=0\right] \\
= & (-1)^{s} \operatorname{det}\left[\begin{array}{cccc}
A_{2,1} & A_{2,3} & \cdots & A_{2, n} \\
A_{3,1} & A_{3,3} & \cdots & A_{3, n} \\
\cdots & \cdots & \cdots & \cdots \\
A_{n, 1} & A_{n, 3} & \cdots & A_{n, n}
\end{array}\right] \\
= & (-1)^{(1+2+\cdots+s)+(s+1+s+2+\cdots+s+s)} \operatorname{det}\left[\begin{array}{cccc}
A_{2,1} & A_{2,3} & \cdots & A_{2, n} \\
A_{3,1} & A_{3,3} & \cdots & A_{3, n} \\
\cdots & \cdots & \cdots & \cdots \\
A_{n, 1} & A_{n, 3} & \cdots & A_{n, n}
\end{array}\right] \\
= & \text { Cofactor of } A_{1,2} .
\end{aligned}
$$

Similarly, we can prove that cofactor of $A_{i, j}$ is equal to cofactor of $A_{i, k}$, for any $i, j, k$. By using the assumption $\sum_{i=1}^{n} A_{i, j}=0$ and applying the above steps to the rows of $A$, we get the cofactor of $A_{i, j}$ equals to cofactor of $A_{k, j}$, for any $i, j, k$.

\section{Resistance matrix with matrix weights}

Let $G$ be a connected weighted graph with $n$ vertices, $m$ edges and all the edge weights of $G$ are positive definite matrices of order $s \times s$. For a positive definite matrix $A$, let $\sqrt{A}$ denote the unique positive definite square root of $A$. The Laplacian matrix $L$ of the graph $G$ is the $n s \times n s$ block matrix whose row and column blocks are index by the vertices of $G$ and $(i, j)^{t h}$ block of $L$ equals to the sum of the inverse of weight matrices of the edges incident with the vertex $i$ if $i=j$, and is equals to $-W^{-1}$ if there is an edge between the vertices $i$ and $j$, where $W$ is the weight of the edge, and zero matrix otherwise. We assign an orientation to each edge of $G$. Then the vertex edge incidence matrix $Q$ of $G$ is the $n s \times m s$ block matrix whose row and column blocks are index by the vertices and the edges of $G$, respectively. The $(i, j)^{t h}$ block of $Q$ is zero matrix if the $i^{t h}$ vertex and the $j^{\text {th }}$ edge are not incident and it is ${\sqrt{W_{j}}}^{-1}$ (respectively, $-{\sqrt{W_{j}}}^{-1}$ ) if the $i^{\text {th }}$ vertex and the $j^{\text {th }}$ edge are incident, and the edge originates (respectively, terminates) at the $i^{\text {th }}$ vertex, where $W_{j}$ is the weight of the $j^{\text {th }}$ edge. In this case, it is easy to see that, the Laplacian matrix $L$ can be written as $L=Q Q^{\prime}$.

Let $L^{\dagger}$ be the Moore-Penrose inverse of the Laplacian matrix $L$ of a graph $G$, and let $K_{i, j}, i, j=$ 
$1,2, \cdots, n$, be the $s \times s$ blocks of $L^{\dagger}$. For the graph $G$, we define the resistance matrix $R$ by an $n \times n$ block matrix whose $(i, j)^{t h}$ block $R_{i, j}$ is defined by

$$
R_{i, j}=K_{i, i}+K_{j, j}-2 K_{i, j}, i, j=1,2, \cdots, n
$$

Since the Moore-Penrose inverse of a matrix always exists and it is unique, so the definition of resistance matrix is well defined. Also, if $s=1$, then the above definition of resistance matrix coincides with the definition of resistance matrix of positive scalar weighted graphs.

In the next theorem, we prove the matrix $L+\frac{1}{n} J \otimes I_{s}$ is nonsingular.

Theorem 3.1. Let $G$ be a connected graph on $n$ vertices such that the edge weights are $s \times s$ positive definite matrices. Then $L+\frac{1}{n} J \otimes I_{s}$ is nonsingular.

Proof. Since each weight matrix is positive definite, we get $L$ is positive semidefinite and rank of $L$ is $n s-s$ [1]. Let the eigenvalues of $L$ be $\lambda_{1}, \lambda_{2}, \cdots, \lambda_{n s-s}, 0,0, \cdots, 0$, where multiplicity of 0 is $s$. Now according to the construction of $L$, we have $L\left(1 \otimes I_{s}\right)=0$. Thus each of the column vector of $1 \otimes I_{s}$, is an eigenvector of $L$ corresponding to the eigenvalue 0 . Note that column vectors of $1 \otimes I_{s}$ are linearly independent. Let $x_{i}$ be an eigenvector of $L$ corresponding to the eigenvalue $\lambda_{i} \neq 0$ for $i=1,2, \cdots, n s-s$. As $L$ is symmetric,

$$
\begin{aligned}
& x_{i}^{\prime}\left(1 \otimes I_{s}\right)=0 \\
\Rightarrow & \left(1^{\prime} \otimes I_{s}\right) x_{i}=0 \\
\Rightarrow & \left(11^{\prime} \otimes I_{s}\right) x_{i}=0 \\
\Rightarrow & \left(J \otimes I_{s}\right) x_{i}=0 .
\end{aligned}
$$

Then

$$
\begin{aligned}
\left(L+\frac{1}{n} J \otimes I_{s}\right)\left(1 \otimes I_{s}\right) & =L\left(1 \otimes I_{s}\right)+\frac{1}{n}\left(J \otimes I_{s}\right)\left(1 \otimes I_{s}\right) \\
& =\frac{1}{n}\left(J 1 \otimes I_{s}\right) \\
& =\left(1 \otimes I_{s}\right) .
\end{aligned}
$$

Thus each of the column vector of $1 \otimes I_{s}$, is an eigenvector of $L+\frac{1}{n} J \otimes I_{s}$ corresponding to the eigenvalue 1 . Now, for each $i=1,2, \cdots, n s-s$,

$$
\begin{aligned}
\left(L+\frac{1}{n} J \otimes I_{s}\right) x_{i} & =L x_{i}+\frac{1}{n}\left(J \otimes I_{s}\right) x_{i} \\
& =L x_{i} \\
& =\lambda_{i} x_{i} .
\end{aligned}
$$

Thus $x_{i}$ is also an eigenvector of $L+\frac{1}{n} J \otimes I_{s}$ corresponding to the eigenvalue $\lambda_{i}$ for $i=1,2, \cdots, n s-s$. Hence the eigenvalues of $L+\frac{1}{n} J \otimes I_{s}$ are $\lambda_{1}, \lambda_{2}, \cdots, \lambda_{n s-s}, 1,1, \cdots, 1$, where multiplicity of 1 is $s$. Thus $L+\frac{1}{n} J \otimes I_{s}$ is invertible. 
In the previous theorem, we have seen that $L+\frac{1}{n} J \otimes I_{s}$ is nonsingular. Now set $X=\left(L+\frac{1}{n} J \otimes I_{s}\right)^{-1}$. Since $L\left(J \otimes I_{s}\right)=\left(J \otimes I_{s}\right) L=\mathbf{0}$, we have

$$
\begin{aligned}
& \left(L+\frac{1}{n} J \otimes I_{s}\right) L=L\left(L+\frac{1}{n} J \otimes I_{s}\right) \\
\Rightarrow & X\left(L+\frac{1}{n} J \otimes I_{s}\right) L X=X L\left(L+\frac{1}{n} J \otimes I_{s}\right) X \\
\Rightarrow & L X=X L .
\end{aligned}
$$

Using this observation, in the next theorem, we derive a formula for the Moore-Penrose inverse of the Laplacian matrix associated with the graph.

Theorem 3.2. Let $G$ be a connected graph on $n$ vertices such that the edge weights are $s \times s$ positive definite matrices and let $L$ be the Laplacian matrix associated with $G$. Then $L^{\dagger}=X-\frac{1}{n} J \otimes I_{s}$.

Proof.

$$
\begin{aligned}
L\left(X-\frac{1}{n} J \otimes I_{s}\right) L & =L X L \\
& =L\left[I_{n s}-\frac{1}{n} X\left(J \otimes I_{s}\right)\right] \\
& =L-\frac{1}{n} L X\left(J \otimes I_{s}\right) \\
& =L-\frac{1}{n} X L\left(J \otimes I_{s}\right) \quad[\text { By (5) }] \\
& =L .
\end{aligned}
$$

Again applying (5), we get

$$
\begin{aligned}
& X\left(J \otimes I_{s}\right)=\left(J \otimes I_{s}\right) X \\
\Rightarrow & X\left(J \otimes I_{s}\right)\left(L+\frac{1}{n} J \otimes I_{s}\right)=\left(J \otimes I_{s}\right) X\left(L+\frac{1}{n} J \otimes I_{s}\right) \\
\Rightarrow & X\left[\frac{1}{n}\left(J \otimes I_{s}\right)\left(J \otimes I_{s}\right)\right]=\left(J \otimes I_{s}\right) \\
\Rightarrow & X\left(J \otimes I_{s}\right)=\left(J \otimes I_{s}\right) .
\end{aligned}
$$

Similarly, we get

$$
\left(J \otimes I_{s}\right) X=\left(J \otimes I_{s}\right)
$$

Now,

$$
\begin{aligned}
\left(X-\frac{1}{n} J \otimes I_{s}\right) L\left(X-\frac{1}{n} J \otimes I_{s}\right) & =X L X \\
& =\left(I_{n s}-\frac{1}{n} X J \otimes I_{s}\right) X \\
& =X-\frac{1}{n} X\left(J \otimes I_{s}\right) X \\
& =X-\frac{1}{n} J \otimes I_{s} \quad[\text { By (7) and (8) }] .
\end{aligned}
$$


Again,

$$
\begin{aligned}
{\left[\left(X-\frac{1}{n} J \otimes I_{s}\right) L\right]^{\prime} } & =L^{\prime}\left(X-\frac{1}{n} J \otimes I_{s}\right)^{\prime} \\
& =L\left(X-\frac{1}{n} J \otimes I_{s}\right) \\
& =L X \\
& =X L \\
& =\left(X-\frac{1}{n} J \otimes I_{s}\right) L
\end{aligned}
$$

Similarly,

$$
\left[L\left(X-\frac{1}{n} J \otimes I_{s}\right)\right]^{\prime}=L\left(X-\frac{1}{n} J \otimes I_{s}\right)
$$

By combining (6), (9), (10) and (11), we get the result.

Now, using Theorem 3.2 and equation (44), for $i, j=1,2, \cdots, n$, we have

$$
R_{i, j}=X_{i, i}+X_{j, j}-2 X_{i, j}
$$

Let $\bar{X}$ denote the diagonal block matrix whose diagonal blocks are $X_{1,1}, X_{2,2}, \cdots, X_{n, n}$. Then, by using the previous equation we get,

$$
R=\bar{X}\left(J \otimes I_{s}\right)+\left(J \otimes I_{s}\right) \bar{X}-2 X
$$

For $i, j=1,2, \cdots, n$, we define the $s \times s$ matrices $\tau_{i}$ and the $n s \times s$ matrix $\tau$ as follows:

$$
\begin{array}{r}
\tau_{i}=2 I_{s}-\sum_{j \sim i} W_{i, j}^{-1} R_{j, i}, \\
\tau=\left[\tau_{1}, \tau_{2}, \cdots, \tau_{n}\right]^{\prime}
\end{array}
$$

Theorem 3.3. Let $G$ be a connected graph on $n$ vertices such that the edge weights are $s \times s$ positive definite matrices and $L$ be the Laplacian matrix associated with $G$. If the matrices $\bar{X}$ and $\tau$ are defined as above, then $L \bar{X}\left(\mathbf{1} \otimes I_{s}\right)+\frac{2}{n}\left(\mathbf{1} \otimes I_{s}\right)=\tau$.

Proof. We have

$$
\left(L+\frac{1}{n} J \otimes I_{s}\right) X=I_{n s}
$$

Equating the $(i, i)^{t h}$ block in both sides, we get

$$
\begin{aligned}
& \sum_{j \sim i} W_{i, j}^{-1} X_{i, i}-\sum_{j \sim i} W_{i, j}^{-1} X_{j, i}+\frac{1}{n} \sum_{j=1}^{n} I_{s} X_{j, i}=I_{s} \\
\Rightarrow & \left(\sum_{j \sim i} W_{i, j}^{-1}\right) X_{i, i}-\sum_{j \sim i} W_{i, j}^{-1} X_{j, i}+\frac{1}{n} \sum_{j=1}^{n} X_{j, i}=I_{s} .
\end{aligned}
$$


Now,

$$
\begin{aligned}
& \left(L+\frac{1}{n} J \otimes I_{s}\right)\left(\mathbf{1} \otimes I_{s}\right)=\mathbf{1} \otimes I_{s} \\
\Rightarrow \quad & X\left(L+\frac{1}{n} J \otimes I_{s}\right)\left(\mathbf{1} \otimes I_{s}\right)=X\left(\mathbf{1} \otimes I_{s}\right) \\
\Rightarrow \quad & \left(\mathbf{1} \otimes I_{s}\right)=X\left(\mathbf{1} \otimes I_{s}\right) \\
\Rightarrow & \left(\mathbf{1}^{\prime} \otimes I_{s}\right)=\left(\mathbf{1}^{\prime} \otimes I_{s}\right) X \\
\Rightarrow & \sum_{j=1}^{n} X_{j, i}=I_{s} \text { for } i=1,2, \cdots, n
\end{aligned}
$$

From (14), we get

$$
\left(\sum_{j \sim i} W_{i, j}^{-1}\right) X_{i, i}-\sum_{j \sim i} W_{i, j}^{-1} X_{j, i}=\left(1-\frac{1}{n}\right) I_{s} .
$$

Now, for $i=1,2, \cdots, n$,

$$
\begin{aligned}
\tau_{i} & =2 I_{s}-\sum_{j \sim i} W_{i, j}^{-1} R_{j, i} \\
& =2 I_{s}-\sum_{j \sim i} W_{i, j}^{-1}\left(X_{i, i}+X_{j, j}-2 X_{j, i}\right) \\
& =\left(1+\frac{1}{n}\right) I_{s}-\sum_{j \sim i} W_{i, j}^{-1} X_{j, j}+\sum_{j \sim i} W_{i, j}^{-1} X_{j, i} . \quad[\mathrm{By}(15)]
\end{aligned}
$$

Let $\gamma_{i}$ be the $i^{\text {th }}$ block of $L \bar{X}\left(\mathbf{1} \otimes I_{s}\right)+\frac{2}{n}\left(\mathbf{1} \otimes I_{s}\right)$. Then, for $i=1,2, \cdots, n$,

$$
\begin{aligned}
\gamma_{i} & =\sum_{k=1}^{n}(L \bar{X})_{i, k} I_{s}+\frac{2}{n} I_{s} \\
& =\sum_{k=1}^{n}\left(\sum_{j=1}^{n} L_{i, j} \bar{X}_{j, k}\right)+\frac{2}{n} I_{s} \\
& =\sum_{k=1}^{n} L_{i, k} \bar{X}_{k, k}+\frac{2}{n} I_{s} \\
& =L_{i, i} \bar{X}_{i, i}+\sum_{k \sim i} L_{i, k} \bar{X}_{k, k}+\frac{2}{n} I_{s} \\
& =\left(\sum_{j \sim i} W_{i, j}^{-1}\right) \bar{X}_{i, i}-\sum_{j \sim i} W_{i, j}^{-1} \bar{X}_{j, j}+\frac{2}{n} I_{s} \\
& =\left(1+\frac{1}{n}\right) I_{s}-\sum_{j \sim i} W_{i, j}^{-1} X_{j, j}+\sum_{j \sim i} W_{i, j}^{-1} X_{j, i} \quad[\mathrm{By}(\underline{15})] \\
& =\tau_{i} .
\end{aligned}
$$


Remark 3.1. Using the previous theorem, we get the following identities:

$$
\begin{aligned}
\left(\mathbf{1}^{\prime} \otimes I_{s}\right) \tau & =\left(\mathbf{1}^{\prime} \otimes I_{s}\right)\left[L \bar{X}\left(\mathbf{1} \otimes I_{s}\right)+\frac{2}{n}\left(\mathbf{1} \otimes I_{s}\right)\right] \\
& =\frac{2}{n}\left(\mathbf{1}^{\prime} \otimes I_{s}\right)\left(\mathbf{1} \otimes I_{s}\right) \\
& =2 I_{s} \\
\Rightarrow \tau^{\prime}\left(\mathbf{1} \otimes I_{s}\right) & =2 I_{s} .
\end{aligned}
$$

Theorem 3.4. Let $G$ be a connected graph on $n$ vertices such that the edge weights are $s \times s$ positive definite matrices and $R$ be the resistance matrix of $G$. Then

$$
\sum_{i=1}^{n} \sum_{j \sim i} W_{i, j}^{-1} R_{j, i}=2(n-1) I_{s} .
$$

Proof. From Theorem 3.2, we have $L^{\dagger}=\left(X-\frac{1}{n} J \otimes I_{s}\right)$. Now,

$$
L L^{\dagger}=L\left(X-\frac{1}{n} J \otimes I_{s}\right)=L X=I_{n s}-\frac{1}{n}\left(J \otimes I_{s}\right) X=I_{n s}-\frac{1}{n} J \otimes I_{s} .
$$

From equation (13), we have

$$
R=\bar{X}\left(J \otimes I_{s}\right)+\left(J \otimes I_{s}\right) \bar{X}-2 X
$$

Thus

$$
\begin{aligned}
L R & =L \bar{X}\left(J \otimes I_{s}\right)-2 L X \\
& =L \bar{X}\left(J \otimes I_{s}\right)-2 I_{n s}+\frac{2}{n}\left(J \otimes I_{s}\right) .
\end{aligned}
$$

Equating the $(i, i)^{\text {th }}$ block in both sides of (17) we get

$$
\begin{aligned}
\sum_{j=1}^{n} L_{i, j} R_{j, i} & =\sum_{j=1}^{n}(L \bar{X})_{i, j}\left(J \otimes I_{s}\right)_{j, i}-2 I_{s}+\frac{2}{n} I_{s} \\
\Rightarrow L_{i, i} R_{i, i}-\sum_{j \sim i} W_{i, j}^{-1} R_{j, i} & =\sum_{j=1}^{n}(L \bar{X})_{i, j} I_{s}-\left(2-\frac{2}{n}\right) I_{s} \\
\Rightarrow-\sum_{j \sim i} W_{i, j}^{-1} R_{j, i} & =\sum_{j=1}^{n} \sum_{k=1}^{n} L_{i, k} \bar{X}_{k, j}-\left(2-\frac{2}{n}\right) I_{s} \\
& =\sum_{j=1}^{n} L_{i, j} \bar{X}_{j, j}-\left(2-\frac{2}{n}\right) I_{s} .
\end{aligned}
$$

Now,

$$
\begin{aligned}
\sum_{i=1}^{n} \sum_{j \sim i} W_{i, j}^{-1} R_{j, i} & =-\sum_{i=1}^{n} \sum_{j=1}^{n} L_{i, j} \bar{X}_{j, j}+\sum_{i=1}^{n}\left(2-\frac{2}{n}\right) I_{s} \\
& =-\sum_{j=1}^{n} \bar{X}_{j, j} \sum_{i=1}^{n} L_{i, j}+2(n-1) I_{s} . \\
& =2(n-1) I_{s} .
\end{aligned}
$$


Using Theorem 3.2, we have the following identity

$$
\begin{aligned}
L R L & =L\left(\bar{X}\left(J \otimes I_{s}\right)+\left(J \otimes I_{s}\right) \bar{X}-2 X\right) L \\
& =-2 L X L \\
& =-2 L\left(L^{\dagger}+\frac{1}{n}\left(J \otimes I_{s}\right)\right) L \\
& =-2 L L^{\dagger} L=-2 L .
\end{aligned}
$$

In the next theorem, we establish the matrix $\tau^{\prime} R \tau$ is positive definite and derive a formula for it.

Theorem 3.5. Let $G$ be a connected graph on $n$ vertices such that the edge weights are $s \times s$ positive definite matrices, and let $L$ and $R$ be the Laplacian matrix and the resistance matrix of $G$, respectively. If the matrices $\bar{X}$ and $\tau$ are defined as in Theorem [3.3, then the matrix $\tau^{\prime} R \tau$ is positive definite and is equal to $2 \bar{x}^{\prime} L \bar{x}+\frac{8}{n}\left[\sum_{i=1}^{n} X_{i i}-I_{s}\right]$, where $\bar{x}=\bar{X}\left(\mathbf{1} \otimes I_{s}\right)$.

Proof. We have,

$$
\begin{aligned}
\tau^{\prime} R \tau= & {\left[\left(\mathbf{1}^{\prime} \otimes I_{s}\right) \bar{X} L+\frac{2}{n}\left(\mathbf{1}^{\prime} \otimes I_{s}\right)\right] R\left[L \bar{X}\left(\mathbf{1} \otimes I_{s}\right)+\frac{2}{n}\left(\mathbf{1} \otimes I_{s}\right)\right] } \\
= & \left(\mathbf{1}^{\prime} \otimes I_{s}\right) \bar{X} L R L \bar{X}\left(\mathbf{1} \otimes I_{s}\right)+\frac{2}{n}\left(\mathbf{1}^{\prime} \otimes I_{s}\right) R L \bar{X}\left(\mathbf{1} \otimes I_{s}\right) \\
& +\frac{2}{n}\left(\mathbf{1}^{\prime} \otimes I_{s}\right) \bar{X} L R\left(\mathbf{1} \otimes I_{s}\right)+\frac{4}{n^{2}}\left(\mathbf{1}^{\prime} \otimes I_{s}\right) R\left(\mathbf{1} \otimes I_{s}\right) .
\end{aligned}
$$

Now,

$$
\begin{aligned}
\left(\mathbf{1}^{\prime} \otimes I_{s}\right) \bar{X} L R L \bar{X}\left(\mathbf{1} \otimes I_{s}\right) & =\left(\mathbf{1}^{\prime} \otimes I_{s}\right) \bar{X}(-2 L) \bar{X}\left(\mathbf{1} \otimes I_{s}\right) \\
& =-2 \bar{x}^{\prime} L \bar{x}
\end{aligned}
$$

and

$$
\begin{aligned}
\frac{2}{n}\left(\mathbf{1}^{\prime} \otimes I_{s}\right) \bar{X} L R\left(\mathbf{1} \otimes I_{s}\right) & =\frac{2}{n}\left(\mathbf{1}^{\prime} \otimes I_{s}\right) \bar{X}\left(L \bar{X}\left(J \otimes I_{s}\right)-2 I_{n s}+\frac{2}{n}\left(J \otimes I_{s}\right)\right)\left(\mathbf{1} \otimes I_{s}\right)[\text { By (17)] } \\
& =2 \bar{x}^{\prime} L \bar{x}
\end{aligned}
$$

Similarly, we can derive the following

$$
\frac{2}{n}\left(\mathbf{1}^{\prime} \otimes I_{s}\right) R L \bar{X}\left(\mathbf{1} \otimes I_{s}\right)=2 \bar{x}^{\prime} L \bar{x} .
$$

Finally,

$$
\begin{aligned}
\frac{4}{n^{2}}\left(\mathbf{1}^{\prime} \otimes I_{s}\right) R\left(\mathbf{1} \otimes I_{s}\right) & =\frac{4}{n^{2}}\left(\mathbf{1}^{\prime} \otimes I_{s}\right)\left(\bar{X}\left(J \otimes I_{s}\right)+\left(J \otimes I_{s}\right) \bar{X}-2 X\right)\left(\mathbf{1} \otimes I_{s}\right) \\
& \left.=\frac{4}{n^{2}}\left[n\left(\mathbf{1}^{\prime} \otimes I_{s}\right) \bar{X}\left(\mathbf{1} \otimes I_{s}\right)+n\left(\mathbf{1}^{\prime} \otimes I_{s}\right) \bar{X}\left(\mathbf{1} \otimes I_{s}\right)-2\left(\mathbf{1}^{\prime} \otimes I_{s}\right) X\left(\mathbf{1} \otimes I_{s}\right)\right)\right] \\
& =\frac{8}{n}\left[\sum_{i=1}^{n} X_{i i}-I_{s}\right] .
\end{aligned}
$$


Then from (19) we get

$$
\tau^{\prime} R \tau=2 \bar{x}^{\prime} L \bar{x}+\frac{8}{n}\left[\sum_{i=1}^{n} X_{i i}-I_{s}\right] .
$$

For $i=1,2, \cdots, n$, the matrices $X_{i i}-\frac{1}{n} I_{s}$ are the diagonal blocks of the matrix $L^{\dagger}$. Since $\sum_{i=1}^{n} X_{i i}-$ $I_{s}=\sum_{i=1}^{n}\left(X_{i i}-\frac{1}{n} I_{s}\right)$, by applying Lemma 2.1, we get the matrices $X_{i i}-\frac{1}{n} I_{s}$ are positive definite for $i=1,2, \cdots, n$. Again, $\bar{x}^{\prime} L \bar{x}=\bar{x}^{\prime} Q Q^{\prime} \bar{x}$ is also positive semi definite matrix. Hence, from (20), we get $\tau^{\prime} R \tau$ is a positive definite matrix.

\section{Determinant, inverse and inertia of the resistance matrix}

In this section, we derive formulae for determinant, inverse and inertia of the resistance matrix.

Consider the partition of the Laplacian matrix $L$ as in (3)

$$
L=\left[\begin{array}{cccc}
L_{11} & L_{12} & \cdots & L_{1 n} \\
L_{21} & L_{22} & \cdots & L_{2 n} \\
\cdots & \cdots & \cdots & \cdots \\
L_{n 1} & L_{n 2} & \cdots & L_{n n}
\end{array}\right]
$$

Then, by definition, we have $\sum_{j=1}^{n} L_{i j}=0$ and $\sum_{i=1}^{n} L_{i j}=0$ for all $i, j \in\{1,2, \cdots, n\}$. By applying Lemma 2.2. we get the cofactors of any two blocks $L_{i j}$ and $L_{k l}$ in $L$ are equal. Using this fact, first we derive a formula for the determinant of the resistance matrix.

Theorem 4.1. Let $G$ be a connected graph on $n$ vertices such that the edge weights are $s \times s$ positive definite matrices, and let $L$ and $R$ be the Laplacian matrix and the resistance matrix of $G$, respectively. Then $\operatorname{det} R=(-1)^{(n-1) s} 2^{(n-3) s} \frac{\operatorname{det}\left(\tau^{\prime} R \tau\right)}{\chi(G)}$, where $\chi(G)$ is the cofactor of any block of $L$.

Proof. By Theorem 3.5, we have $\tau^{\prime} R \tau$ is a nonsingular matrix. Using the Schur formula for the determinant, we have

$$
\begin{aligned}
\operatorname{det}\left[\begin{array}{cc}
-\frac{1}{2} L & \tau \\
\tau^{\prime} & -\tau^{\prime} R \tau
\end{array}\right] & =\operatorname{det}\left(-\tau^{\prime} R \tau\right) \operatorname{det}\left(-\frac{1}{2} L+\tau\left(\tau^{\prime} R \tau\right)^{-1} \tau^{\prime}\right) \\
& =\operatorname{det}\left(-\tau^{\prime} R \tau\right) \operatorname{det} R^{-1} \\
& =(-1)^{s} \operatorname{det}\left(\tau^{\prime} R \tau\right) \operatorname{det} R^{-1}
\end{aligned}
$$

Using Remark 3.1, adding all the column blocks except the last column block to the first column block and adding all the row blocks except the last row block to the first row block of the matrix 


$$
\begin{aligned}
& {\left[\begin{array}{cc}
-\frac{1}{2} L & \tau \\
\tau^{\prime} & -\tau^{\prime} R \tau
\end{array}\right] \text {, we get }} \\
& \operatorname{det}\left[\begin{array}{c|c}
-\frac{1}{2} L & \tau \\
\hline \tau^{\prime} & -\tau^{\prime} R \tau
\end{array}\right]=\operatorname{det}\left[\begin{array}{c|c|c}
\mathbf{0}_{s \times s} & \mathbf{0}_{s \times(n-1) s} & 2 I_{s} \\
\hline \mathbf{0}_{(n-1) s \times s} & -\frac{1}{2} L(1,1) & * \\
\hline 2 I_{s} & * & -\tau^{\prime} R \tau
\end{array}\right] \text {, } \\
& \text { where } L(1,1) \text { is the cofactor of } L_{1,1} \text { in } L \text {. } \\
& =(-1)^{(1+2+\cdots+s)+(n s+1+n s+2+\cdots+n s+s)} \operatorname{det}\left(2 I_{s}\right) \operatorname{det}\left[\begin{array}{c|c}
\mathbf{0}_{(n-1) s \times s} & -\frac{1}{2} L(1,1) \\
\hline 2 I_{s} & *
\end{array}\right] \text {, } \\
& \text { by expanding Laplace expansion. } \\
& =(-1)^{n s^{2}} 2^{s} \operatorname{det}\left[\begin{array}{c|c}
\mathbf{0}_{(n-1) s \times s} & -\frac{1}{2} L(1,1) \\
\hline 2 I_{s} & *
\end{array}\right] \\
& =(-1)^{n s^{2}} 2^{s}(-1)^{(n-1) s^{2}} \operatorname{det}\left(2 I_{s}\right) \operatorname{det}\left(-\frac{1}{2} L(1,1)\right) \\
& =\frac{(-1)^{n s}}{2^{(n-3) s}} \operatorname{det} L(1,1) \\
& =\frac{(-1)^{n s}}{2^{(n-3) s}} \chi(G) \text {. }
\end{aligned}
$$

Using this in (22) we get

$$
\operatorname{det} R=(-1)^{(n-1) s} 2^{(n-3) s} \frac{\operatorname{det}\left(\tau^{\prime} R \tau\right)}{\chi(G)}
$$

In the next theorem, we establish that the resistance matrices are nonsingular and derive a formula for the inverse of them.

Theorem 4.2. Let $G$ be a connected graph on $n$ vertices such that the edge weights are $s \times s$ positive definite matrices, and let $L$ and $R$ be the Laplacian matrix and the resistance matrix of $G$, respectively. Then $R$ is nonsingular and $R^{-1}=-\frac{1}{2} L+\tau\left(\tau^{\prime} R \tau\right)^{-1} \tau^{\prime}$.

Proof. From equation (17), we have

$$
\begin{aligned}
L R & =L \bar{X}\left(J \otimes I_{s}\right)-2 I_{n s}+\frac{2}{n} J \otimes I_{s} \\
\Rightarrow L R+2 I_{n s} & =\left[L \bar{X}\left(\mathbf{1} \otimes I_{s}\right)+\frac{2}{n}\left(\mathbf{1} \otimes I_{s}\right)\right]\left(\mathbf{1}^{\prime} \otimes I_{s}\right) \\
& =\tau\left(\mathbf{1}^{\prime} \otimes I_{s}\right)[\text { By Lemma 3.3] } \\
\Rightarrow\left(L R+2 I_{n s}\right) \tau & =\tau\left(\mathbf{1}^{\prime} \otimes I_{s}\right) \tau \\
& =2 \tau[\text { By Remark 3.1] } \\
\Rightarrow L R \tau & =0 .
\end{aligned}
$$

From Theorem 3.5. we have $\tau^{\prime} R \tau$ is a positive definite matrix, so $R \tau$ is nonzero. Since $L$ has exactly $s$ number of zero eigenvalues and $L\left(1 \otimes I_{s}\right)=0$, the column space of $R \tau$ is a subspace of column space 
of $\left(1 \otimes I_{s}\right)$. Thus, there exist a matrix $C$ such that

$$
\begin{aligned}
R \tau & =\left(1 \otimes I_{s}\right) C \\
\Rightarrow \tau^{\prime} R \tau & =\tau^{\prime}\left(\mathbf{1} \otimes I_{s}\right) C \\
& =2 C \quad[\text { By Remark 3.1] } \\
\Rightarrow C & =\frac{1}{2} \tau^{\prime} R \tau .
\end{aligned}
$$

Now,

$$
\begin{aligned}
R \tau & =\frac{1}{2}\left(\mathbf{1} \otimes I_{s}\right) \tau^{\prime} R \tau \\
\Rightarrow \tau^{\prime} R & =\frac{1}{2} \tau^{\prime} R \tau\left(\mathbf{1}^{\prime} \otimes I_{s}\right)
\end{aligned}
$$

and

$$
\begin{aligned}
\left(-\frac{1}{2} L+\tau\left(\tau^{\prime} R \tau\right)^{-1} \tau^{\prime}\right) R & =-\frac{1}{2} L R+\tau\left(\tau^{\prime} R \tau\right)^{-1} \tau^{\prime} R \\
& =-\frac{1}{2} L R+\frac{1}{2} \tau\left(\tau^{\prime} R \tau\right)^{-1} \tau^{\prime} R \tau\left(\mathbf{1}^{\prime} \otimes I_{s}\right) \quad[\text { By (25) }] \\
& =-\frac{1}{2} L R+\frac{1}{2} \tau\left(\mathbf{1}^{\prime} \otimes I_{s}\right) \\
& =I_{n s} .[\text { By }(\underline{23})]
\end{aligned}
$$

Thus, the matrix $R$ is nonsingular, and $R^{-1}=-\frac{1}{2} L+\tau\left(\tau^{\prime} R \tau\right)^{-1} \tau^{\prime}$.

Remark 4.1. Using (23) we get

$$
\begin{aligned}
& L R=\tau\left(\mathbf{1}^{\prime} \otimes I_{s}\right)-2 I_{n s} \\
\Rightarrow & L R Q=\tau\left(\mathbf{1}^{\prime} \otimes I_{s}\right) Q-2 Q \\
\Rightarrow & Q Q^{\prime} R Q=-2 Q \quad\left[\text { As }\left(\mathbf{1}^{\prime} \otimes I_{s}\right) Q=\mathbf{0}\right] \\
\Rightarrow & Q^{\prime} R Q=-2 I_{(n-1) s} . \quad[\text { As } Q \text { has full column rank }]
\end{aligned}
$$

Interlacing inequality for the eigenvalues of distance and Laplace matrices of a matrix weighted tree is given in [1]. Using the fact $Q^{\prime} R Q=-2 I_{(n-1) s}$ we can similarly prove the following:

Theorem 4.3. Let $G$ be a weighted graph on $n$ vertices, where each weight is a positive definite matrix of order s. Let $R$ be the resistance matrix of $G$ and $L$ denote the Laplacian matrix of $G$. Let $\mu_{1} \geq \mu_{2} \geq \cdots \geq \mu_{n s}$ be the eigenvalues of $R$ and $\lambda_{1} \geq \lambda_{2} \geq \cdots \geq \lambda_{n s-s}>\lambda_{n s-s+1}=\cdots=\lambda_{n s}=0$ be the eigenvalues of $L$. Then

$$
\mu_{s+i} \leq-\frac{2}{\lambda_{i}} \leq \mu_{i} \quad \text { for } i=1,2, \cdots, n s-s
$$

For an $n \times n$ symmetric matrix $A$, let $p_{+}(A), p_{-}(A)$ and $p_{0}(A)$ denote the number of positive, negative and zero eigenvalues of $\mathrm{A}$, respectively. Then 3 -tuple $\left(p_{+}(A), p_{-}(A), p_{0}(A)\right)$ is called the inertia of the matrix $A$.

Next we derive the formula for the inertia of the resistance matrix of a graph with matrix weights. 
Theorem 4.4. Let $G$ be a weighted graph on $n$ vertices, where each weight is a positive definite matrix of order $s$ and $R$ be the resistance matrix of $G$. Then inertia of $R$ is $(s, n s-s, 0)$.

Proof. Let $\mu_{1} \geq \mu_{2} \geq \cdots \geq \mu_{n s}$ be the eigenvalues of $R$. Using the previous theorem we have $\mu_{s+i} \leq 0$, for all $i=1,2, \cdots,(n-1) s$. Again in the matrix $R, 0_{s \times s}$ is a principal submatrix. Since $R$ is nonsingular, using interlacing theorem, we have $\mu_{i} \geq 0$, for all $i=1,2, \cdots, s$. Hence the inertia of $R$ is $(s, n s-s, 0)$.

Acknowledgement:The second author acknowledges the support of the JC Bose Fellowship, Department of Science Technology, Government of India. M. Rajesh Kannan would like to thank Department of Science and Technology for the financial support.

\section{References}

[1] Fouzul Atik, M. Rajesh Kannan, and R.B. Bapat, On distance and Laplacian matrices of trees with matrix weights, arXiv:1710.10097.

[2] R. B. Bapat, Resistance matrix of a weighted graph, MATCH Commun. Math. Comput. Chem. (2004), no. 50, 73-82. MR 2037425

[3] _ Determinant of the distance matrix of a tree with matrix weights, Linear Algebra Appl. 416 (2006), no. 1, 2-7. MR 2232916

[4] Ravindra B. Bapat, Graphs and matrices, second ed., Universitext, Springer, London; Hindustan Book Agency, New Delhi, 2014. MR 3289036

[5] R.B. Bapat, S. J. Kirkland, and M. Neumann, On distance matrices and Laplacians, Linear Algebra Appl. 401 (2005), 193-209. MR 2133282

[6] R. L. Graham and L. Lovász, Distance matrix polynomials of trees, Adv. in Math. 29 (1978), no. 1, 60-88. MR 0480119

[7] R. L. Graham and H. O. Pollak, On the addressing problem for loop switching, Bell System Tech. J. 50 (1971), 2495-2519. MR 0289210

[8] Roger A. Horn and Charles R. Johnson, Topics in matrix analysis, Cambridge University Press, Cambridge, 1994, Corrected reprint of the 1991 original. MR 1288752

[9] Douglas J Klein and Milan Randić, Resistance distance, Journal of mathematical chemistry 12 (1993), no. 1, 81-95.

[10] Jack H. Koolen, Greg Markowsky, and Jongyook Park, On electric resistances for distance-regular graphs, European J. Combin. 34 (2013), no. 4, 770-786. MR 3010117 
[11] Xiaogang Liu, Jiang Zhou, and Changjiang Bu, Resistance distance and Kirchhoff index of $R$ vertex join and R-edge join of two graphs, Discrete Appl. Math. 187 (2015), 130-139. MR 3332822

[12] Russell Merris, The distance spectrum of a tree, J. Graph Theory 14 (1990), no. 3, 365-369. MR 1060864

[13] Lizhu Sun, Wenzhe Wang, Jiang Zhou, and Changjiang Bu, Some results on resistance distances and resistance matrices, Linear Multilinear Algebra 63 (2015), no. 3, 523-533. MR 3273992

[14] Wenjun Xiao and Ivan Gutman, On resistance matrices, MATCH Commun. Math. Comput. Chem. (2003), no. 49, 67-81. MR 2019257

[15] _ Resistance distance and Laplacian spectrum, Theoretical Chemistry Accounts 110 (2003), no. 4, 284-289.

[16] Jiang Zhou, Zhongyu Wang, and Changjiang Bu, On the resistance matrix of a graph, Electron. J. Combin. 23 (2016), no. 1, Paper 1.41, 10. MR 3484746 